\title{
Stress-Activated Protein Kinase OsSAPK9 Regulates Tolerance to Salt Stress and Resistance to Bacterial Blight in Rice
}

\author{
Fan Zhang ${ }^{1,2 \dagger}$, Dan Zeng ${ }^{1 \dagger}$, Liyu Huang ${ }^{1,3}$, Yingyao Shi ${ }^{4}$, Tengjun Chen ${ }^{4}$, Fan Zhang ${ }^{1 *}$ and Yongli Zhou ${ }^{1 *}$ (D
}

\begin{abstract}
Background: Salt stress and bacterial blight caused by Xanthomonas oryzae pv. oryzae (Xoo) are key limiting factors of rice (Oryza sativa L.) yields. Members of sucrose non-fermenting 1 (SNF1)-related protein kinase 2 (SnRK2), which is a family of plant-specific Ser/Thr kinases, are important components of signaling pathways involved in plant developmental processes and responses to stresses. There are 10 members of the SnRK2 family in rice; however, their functions are poorly understood, as are the underlying molecular mechanisms.

Results: In this study, we found that OsSAPK9, which belongs to the SnRK2 family, positively regulated salt-stress tolerance and strain-specific resistance to bacterial blight in rice. RNA sequencing revealed that there were 404 and 1324 genes differentially expressed in OSSAPK9-RNAi in comparison with wild-type plants under salt-stress conditions and after $X_{0}$ inoculation, respectively, which participate in basic metabolic processes. In total, 65 common differentially expressed genes involved mainly in defense responses were detected both under salt-stress conditions and after Xoo inoculation. Moreover, in vivo and in vitro experiments demonstrated that OsSAPK9 forms a protein complex with the molecular chaperones OsSGT1 and OsHsp90, and transgenic plants overexpressing OsSGT1 exhibited decreased tolerances to salt stress and significantly increased resistance levels to bacterial blight. Thus, OsSAPK9 may function as a center node regulator of salt-stress responses and disease-resistance pathways through its interaction with OsSGT1 in rice.
\end{abstract}

Conclusion: This study confirms that OsSAPK9 functions as a positive regulator of salt-stress responses and disease resistance through its interaction with OsSGT1 in rice.

Keywords: Rice, The SnRK2 family, OsSAPK9, Salt stress, Bacterial blight, OsSTG1

\section{Background}

Rice (Oryza sativa L.) is an important staple food crop for more than half the global population. The large worldwide area for rice cultivation has led to its growth in diverse ecosystems in which it is exposed to diverse stresses. Soil salinization and bacterial blight caused by Xanthomonas oryzae pv. oryzae (Xoo) are two of the main constraints that lead to significant yield losses in rice growing regions in China and South/Southeast Asia (Nino-Liu et al. 2006). Plants have developed

\footnotetext{
*Correspondence: zhangfan03@caas.cn; zhouyongli@caas.cn

${ }^{\dagger}$ Fan Zhang and Dan Zeng contributed equally to this work.

${ }^{1}$ Institute of Crop Sciences/National Key Facility for Crop Gene Resources and Genetic Improvement, Chinese Academy of Agricultural Sciences, 12 South

Zhong-Guan-Cun Street, Beijing 100081, China

Full list of author information is available at the end of the article
}

sophisticated mechanisms to respond to environmental stresses (Fujita et al. 2006; Sharma et al. 2013). Identifying the key components in the signaling pathways involved in these stress responses will provide information needed to breed tolerance to multiple stresses and improve rice yields. Members of sucrose non-fermenting 1 (SNF1)-related protein kinase 2 (SnRK2), which is a family of plant-specific Ser/Thr kinases, are important components of the signaling pathways involved in abscisic acid (ABA)-dependent developmental processes and responses to abiotic stresses (Fujii et al. 2011; Kulik et al. 2011; Yan et al. 2017). There are 10 known SnRK2 genes in Arabidopsis and 11, 8, and 20 known SnRK2 genes in maize, potato, and cotton, respectively (Bai et al. 2017; Huai et al. 2008; Liu et al. 2017; Saha et al. 2014). The functions of SnRK2s in Arabidopsis thaliana have been 
widely studied. AtSnRK2s not only function in several developmental processes and responses to saline and drought (Cheng et al. 2017; Grondin et al. 2015; Kim et al. 2012; McLoughlin et al. 2012; Soma et al. 2017; Tan et al. 2018; Yoshida et al. 2002; Zheng et al. 2010), but they are also involved in disease resistance. For example, AtSnRK2.8 mediates phosphorylation and salicylic acid signals, which coordinately function to activate NPR1 through a dual-step process that leads to systemic immunity (Lee et al. 2015). At present, 10 members of the SnRK2 family have been identified in rice and are designated stress-activated protein kinases1-10 (OsSAPK1-10) (Kobayashi et al. 2004). The expression levels of all 10 OSSAPKs are up-regulated under saltstress conditions. The overexpression of OsSAPK4 increases the tolerance to oxidative stresses (Diédhiou et al. 2008), while the overexpression SAPK9 from Oryza rufipogon in a drought-sensitive rice line enhances drought tolerance and yield-related traits (Dey et al. 2016). Conversely, sapk2 mutants are more sensitive to drought stress than wild-type (WT) plants (Lou et al. 2017). OsSAPK3, OsSAPK5, OsSAPK7, and OsSAPK9 are up-regulated when the transgenic rice line carrying the heterologous resistance gene $R \times o 1$ is inoculated with Xanthomonas oryzae pv. oryzicola (Xu et al. 2013), while OsSAPK2 knock-down mutants increase the susceptibility to bacterial blight ( $\mathrm{Hu}$ et al. 2015). However, while OsSAPKs might be associated with responses to abiotic and biotic stresses in rice, the functions of this gene family are poorly understood and their underlying molecular mechanisms have yet to be elucidated. In the present study, we used OsSAPK9-RNAi and OsSAPK9overexpression transgenic lines to show that OSSAPK9 is involved in tolerance to salt stress and resistance to bacterial blight. We also showed that OsSAPK9 interacts with OsSGT1 to regulate these processes. Additionally, we used transcriptome profiling to investigate the defense responses to salt stress and Xoo infection mediated by OsSAPK9.

\section{Results}

OsSAPK9 Expression in Rice in Response to Salt Stress and Xoo Infection

Each of the 10 members of the rice SnRK2 family, including OSSAPK9, are activated by hyperosmotic stress in cultured cell protoplasts (Kobayashi et al. 2004). Here, we demonstrated that OSSAPK9's expression was rapidly induced $2 \mathrm{~h}$ after rice seedlings were treated with 100 $\mathrm{mM} \mathrm{NaCl}$ (Additional file 1: Figure S1a). In addition, OsSAPK9's transcription levels in rice plants increased more than 3-fold at $6 \mathrm{~h}$ and $72 \mathrm{~h}$ after inoculation with the Xoo strain GD1358 (Additional file 1: Figure S1b). Thus, OSSAPK9 may be up-regulated in response to salt stress and Xoo infection.

\section{OsSAPK9 Positively Regulates Tolerance to Salt Stress in} Rice

To determine the biological function of OsSAPK9, OsSAPK9-RNAi (denoted with Ri) and OsSAPK9-overexpression (denoted with $\mathrm{OE}$ ) transgenic rice lines were generated (Additional file 2: Figure S2). The phenotypic responses of OsSAPK9-RNAi, OsSAPK9-OE, and WT plants to salt stress were examined. OsSAPK9-RNAi lines Ri-21 and Ri-27 were more sensitive to salt stress in comparison with the WT, and the survival rates of Ri-21 and Ri-27 were significantly reduced $7 \mathrm{~d}$ after treatment with $100 \mathrm{mM} \mathrm{NaCl}$ (Fig. 1a, c). OsSAPK9-OE lines OE1 and OE2 were more tolerant to the salt treatment, and their survival rates significantly increased in comparison with the WT (Fig. 1b, g). To examine the physiological changes in salt-stressed OsSAPK9-RNA $i$ and OsSAPK9-OE lines, we measured known physiological parameters that are associated with salt stress. The accumulation of malondialdehyde (MDA) increased considerably under salt-stress conditions in shoots of OsSAPK9-RNA $i$, OsSAPK9-OE, and WT plants compared with under normal conditions (Fig. 1d, h). When plants were treated with $\mathrm{NaCl}$, the MDA contents were significantly higher in the OsSAPK9-RNAi than in the WT plants and significantly lower in OSSAPK9-OE plants than in the WT plants (Fig. 1d, h). After treatment with $100 \mathrm{mM} \mathrm{NaCl}$, the peroxidase (POD) and catalase (CAT) activities in the OsSAPK9-RNAi plants were significantly lower than those in the WT plants (Fig. 1e, f). Thus, OsSAPK9 positively regulates rice tolerance to salt stress. The shoots of OsSAPK9-RNAi lines Ri-21 and Ri-27 were shorter than those of the WT plants after treatments with $50 \mu \mathrm{m} \mathrm{NaCl}$; however, there were no significantly differences in the root lengths of these plants (Additional file 3: Figure S3a, b). The shoot dry weights and root dry weights of the Ri-21 and Ri-27 lines were significantly less than those of the WT plants (Additional file 3: Figure S3c). Thus, the suppression of OsSAPK9 appears to significantly decrease salt tolerance in rice.

\section{OsSAPK9 Increases Strain-Specific Resistance to Bacterial Blight in Rice}

To investigate whether OSSAPK9 confers tolerance to bacterial blight in rice, OsSAPK9-RNAi, OsSAPK9-OE, and WT plants were separately inoculated with four Xoo strains (GD1358, JS97-2, PXO340, and PXO347). When the OSSAPK9-RNAi transgenic lines were inoculated with GD1358, the lesion lengths (LLs) were significantly longer than those of the WT plants and the bacterial populations were significantly increased (Fig. $2 \mathrm{a}-\mathrm{c}$ ). In contrast, when the OSSAPK9-OE transgenic lines were inoculated with GD1358, the LLs were significantly shorter than those of the WT plants and the bacterial growth was reduced (Fig. 2d-f). OsSAPK9-RNAi and OsSAPK9-OE transgenic lines inoculated with PXO347 showed similar responses to those observed following 


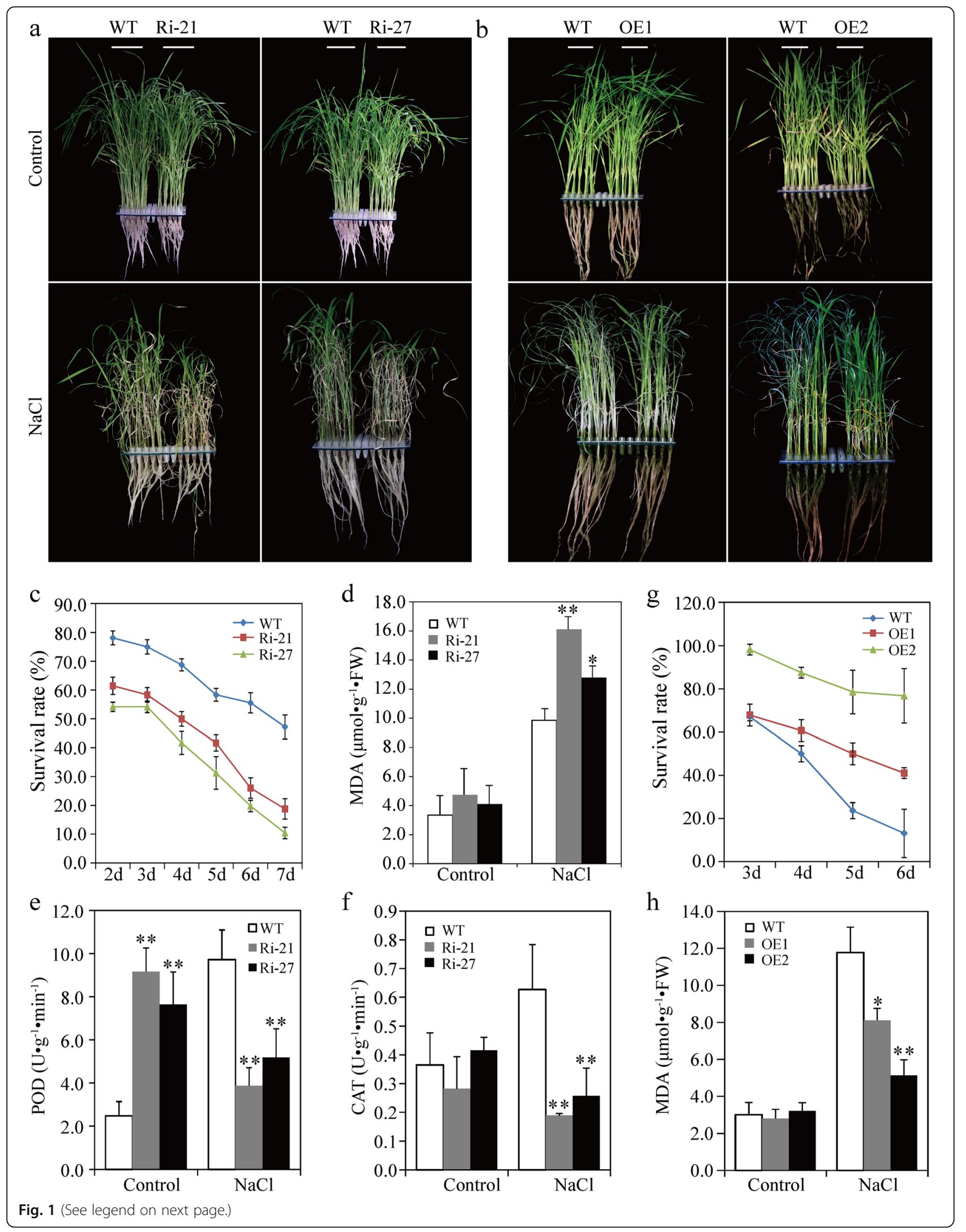


(See figure on previous page.)

Fig. 1 Phenotypic responses of OsSAPK9-RNAi, OsSAPK9-overexpression (OSSAPK9-OE), and wild-type (WT) plants to salt-stress conditions. a Phenotypes of OsSAPK9-RNAi and WT plants treated with or without $100 \mathrm{mM} \mathrm{NaCl}$. Photographs were taken $6 \mathrm{~d}$ after treatment. $\mathbf{b}$ Phenotypes of OsSAPK9-OE and WT plants treated with or without $100 \mathrm{mM} \mathrm{NaCl}$. Photographs were taken $6 \mathrm{~d}$ after treatment.c Survival rates of OsSAPK9-RNAi and WT plants under salt-stress conditions (100 mM NaCl). d Malondialdehyde (MDA) contents in OsSAPK9-RNAi and WT plants at $6 \mathrm{~d}$ after being treated with $100 \mathrm{mM} \mathrm{NaCl}$. e Peroxidase (POD) and (f) Catalase (CAT) activities in OsSAPK9-RNAi and WT plants $6 \mathrm{~d}$ after being treated with 100 $\mathrm{mM} \mathrm{NaCl}$. g Survival rates of OSSAPK9-OE and WT plants under salt-stress conditions (100 mM NaCl). $\mathbf{h}$ MDA contents in OsSAPK9-OE and WT plants $6 \mathrm{~d}$ after being treated with $100 \mathrm{mM} \mathrm{NaCl}$. Values are presented as the means and standard errors of three replicates. ${ }^{*} P<0.05$, according to a Student's t-test

GD1358 inoculation (Additional file 4: Figure S4a, b, g, h). However, no significant differences were detected between the LLs of both the OsSAPK9-RNAi and OSSAPK9-OE transgenic lines and the WT plants when Xoo strains PXO340 and JS97-2 were tested (Additional file 4: Figure S4c-f, i-l). Thus, OsSAPK9's overexpression may positively regulate strain-specific resistance to bacterial blight in rice.

\section{Interaction Between OsSAPK9 and the Molecular Chaperone Protein OsSGT1}

To identify any proteins that interact with OsSAPK9, the OSSAPK9 protein-coding sequence (CDS) was used as bait to screen a rice-Xoo cDNA library using a yeast two-hybrid system. We identified 31 potential interacting molecules (Additional file 8: Table S1), including OsSGT1, which encodes a protein involved in disease resistance (Austin et al. 2002; Azevedo et al. 2002). To verify the interaction between OsSAPK9 and OsSGT1, we conducted a yeast two-hybrid, glutathione S-transferase (GST) pull-down, bimolecular fluorescence complementation (BiFC), and co-immunoprecipitation (Co-IP) assays. Yeast cells co-transformed with the constructs encoding AD-OsSGT1 and BD-OsSAPK9, or BD-53 and $\mathrm{AD}-\mathrm{T}$ (the positive controls), were able to grow in synthetic dextrose $(\mathrm{SD})^{4-}$ dropout medium (lacking Leu, Trp, His, and Ade). In contrast, yeast cells co-expressing

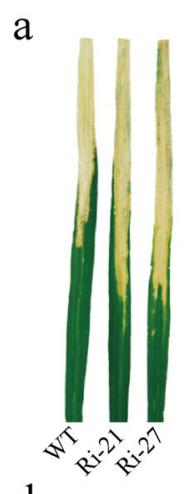

d

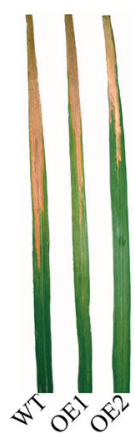

\section{b}
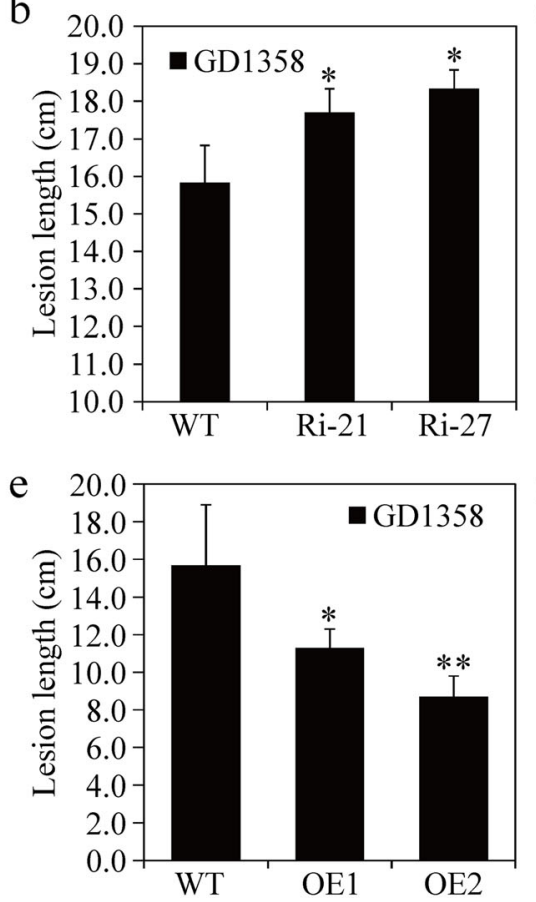

$\mathrm{C}$
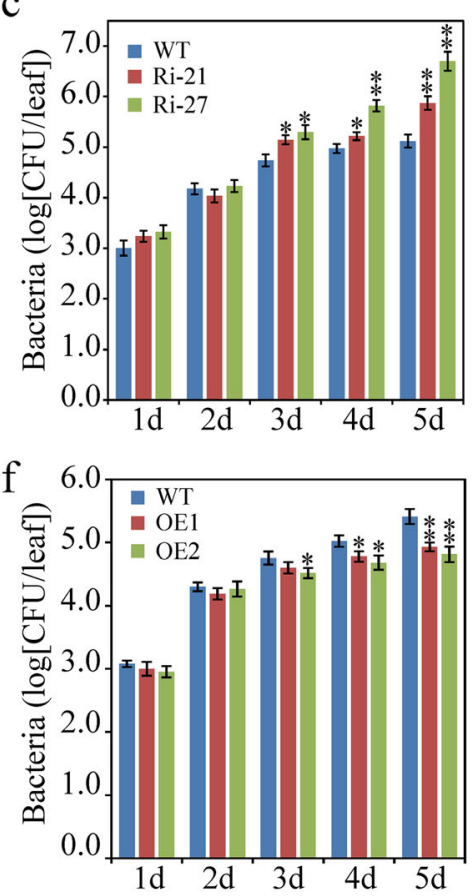

Fig. 2 Phenotypic responses of OSSAPK9-RNAi, OSSAPK9-overexpression (OSSAPK9-OE), and wild-type (WT) plants to infection by Xanthomonas oryzae pv. oryzae (Xoo) strain GD1358. a Phenotypes of OsSAPK9-RNAi and WT plants infected with Xoo strain GD1358. Photographs were taken 12 $\mathrm{d}$ after inoculation. $\mathbf{b}$ Lesion lengths of bacterial blight in the OsSAPK9-RNAi and WT plants $12 \mathrm{~d}$ after inoculation with XoO strain GD1358. c Growth curves of the Xoo strain GD1358 in OsSAPK9-RNAi and WT plants. CFU, colony-forming units. d Phenotypes of OsSAPK9-OE and WT plants infected with XoO strain GD1358. Photographs were taken $12 \mathrm{~d}$ after inoculation. e Lesion lengths of bacterial blight in the OsSAPK9-OE and WT plants $12 \mathrm{~d}$ after inoculation with XOO strain GD1358. f Growth curves of XOO strain GD1358 in OsSAPK9-OE and WT plants. CFU, colony-forming units. Values are presented as the means and standard errors of three replicates. ${ }^{*} P<0.05$, according to a Student's $t$-test 
the constructs encoding $\mathrm{AD}$ and $\mathrm{BD}-\mathrm{OsSAPK}$, or $\mathrm{BD}$ and AD-OsSGT1 (the negative controls), were unable to grow in the $\mathrm{SD}^{4-}$ drop-out medium (Fig. 3a). When the His-OsSAPK9 fusion protein and the His protein control were incubated with recombinant GST-OsSGT1 and GST, respectively, only His-OsSAPK9 was able to bind GST-OsSGT1, indicating that there is a direct interaction between OsSAPK9 and OsSGT1 (Fig. 3b; Additional file 5: Figure S5a). To determine whether OsSAPK9 interacts with OsSGT1 in vivo, we conducted BiFC assays using Nicotiana benthamiana plants. In leaves co-expressing $p N Y F P-O s S A P K 9$ and $p C C F P$ OsSGT1, BiFC signals were detected in the cytoplasm and nucleus (Fig. 3c). Co-IP assays using N. benthamiana plants expressing green fluorescent protein (GFP)OsSAPK9 and/or MYC-OsSGT1 revealed that the antiGFP antibodies pulled down MYC-OsSGT1. The signals were only detectable in the GFP-OsSAPK9 and MYCOsSGT1 co-expression leaves (Fig. 3d), confirming the interaction between OsSAPK9 and OsSGT1. The tetratricopeptide repeat (TPR), CHORD-containing protein and SGT1 (CS), and SGT-specific (SGS) domains form three distinct regions of SGT1 (Azevedo et al. 2002; Shirasu 2009). The yeast two-hybrid analysis revealed that OsSAPK9 interacted with the TPR, central CS, deleted TPR domain $(\triangle T P R)$, and deleted SGS domain $(\triangle \mathrm{SGS})$ regions of OsSGT1, while there was no interaction between the C-terminal SGS region of OsSGT1 and OsSAPK9 (Fig. 4a, b). Consistent with these observations, the pull-down and $\mathrm{BiFC}$ assays confirmed that OsSAPK9 interacted with the TPR and CS domains of OsSGT1 (Fig. 4c-e; Additional file 5: Figure S5b, c). SGT1 interacts with Hsp90 to promote protein folding and stability in plants (Meldau et al. 2011). In this study, BiFC signals in the cytoplasm and the nucleus were detected in $N$. benthamiana plants co-transformed with the $p N Y F P-O s S A P K 9$ and $p C C F P-O s H s p 90$ vectors (Fig. 4f). Additionally, we found that GST-OsHsp90 bound to His-OsSAPK9 in pull-down assays, while neither GST nor His bound to His-OsSAPK9 or GSTOsHsp90 (Fig. 4g; Additional file 5: Figure S5d). These results suggest that OsSAPK9, OsSGT1, and OsHsp90 together form a protein complex.

\section{OsSGT1 Regulates Rice Responses to Salt Stress and Bacterial Blight}

To functionally characterize the interaction between OsSAPK9 and OsSGT1, the responses of OsSGT1-overexpression (denoted with SA) transgenic rice lines (SA24 and SA28) to salt stress and Xoo inoculation were investigated (Additional file 6: Figure S6). After $10 \mathrm{~d}$ of treatment with $100 \mathrm{mM} \mathrm{NaCl}$, the survival rates of the SA24 and SA28 lines were $13.3 \%$ and $38.0 \%$, respectively. These rates were significantly lower than that of the WT plants (67.5\%; Fig. 5a, b). Furthermore, the MDA contents were significantly greater in OSSGT1-OE lines than a

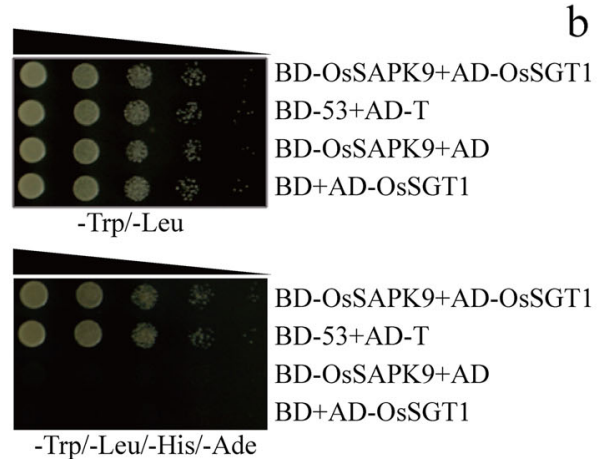

c

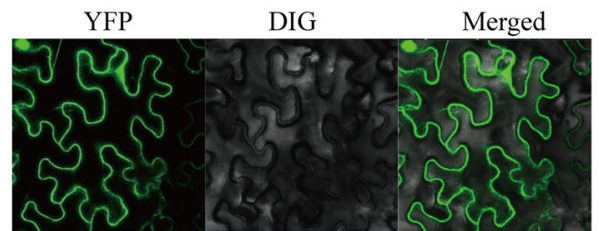

b

His-OsSAPK $9 \quad 10 \%+\quad+\quad+$

GST-OsSGT1 - -++

$\begin{array}{rllll}\text { His } & - & - & + & - \\ \text { GST } & - & + & - & -\end{array}$
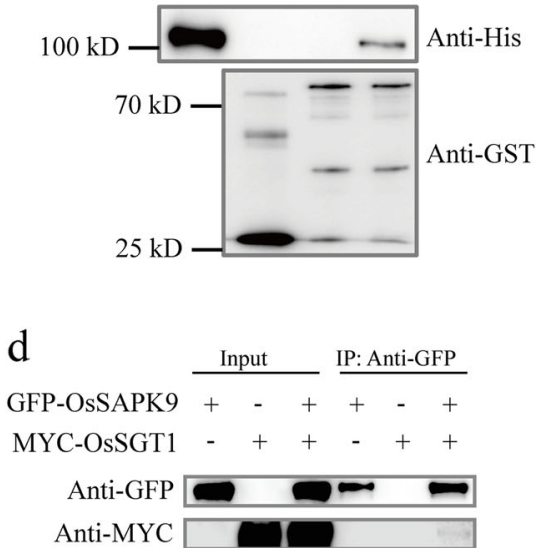

Fig. 3 OsSAPK9 interactions with OsSGT1 in vitro and in vivo. a OSSAPK9 interaction with OsSGT1 in yeast. AH109 yeast transformants diluted 10, 100, 1000, or 10,000 times were plated on synthetic dextrose (SD) medium lacking Trp and Leu amino acids (SD - L/T) or SD medium lacking Trp, Leu, His, and Ade amino acids (SD - L/T/W/A). b OsSAPK9 bound to OsSGT1 in the glutathione S-transferase (GST) pull-down assay. c OsSAPK9 interaction with OsSGT1 in Nicotiana benthamiana cells in the bimolecular fluorescence complementation assay. OsSAPK9 was fused to an Nterminal yellow fluorescent protein (NYFP-OsSAPK9) and OsSGT1 was fused to a C-terminal cyan fluorescent protein (CCFP-OsSGT1). d OsSAPK9 bound to OsSGT1 in the co-immunoprecipitation assay. Constructs encoding green fluorescent protein-tagged OsSAPK9 and Myc-tagged OsSGT1 were co-expressed in Agrobacterium-infiltrated N. benthamiana cells. Tissues were harvested $3 \mathrm{~d}$ after infiltration 


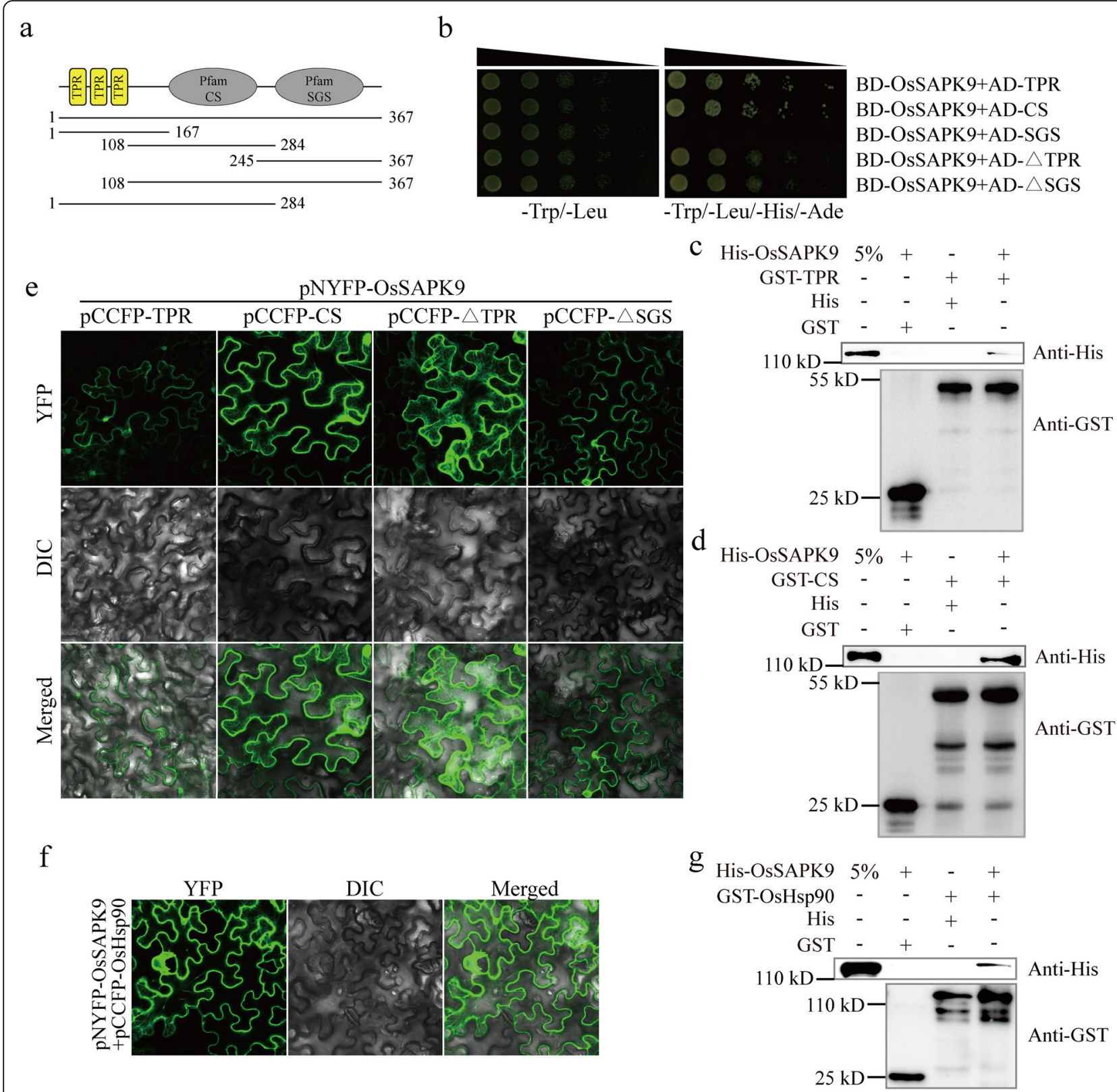

Fig. 4 OsSAPK9 interactions with different OsSGT1 domains in vitro and in vivo. a Schematic of the functional domain of OsSGT1. Positions of the tetratricopeptide repeat (TPR) domain (1-167 amino acids), the CHORD-containing protein and SGT1 (CS) domain (108-284 amino acids), the SGT-specific (SGS) domain (245-367 amino acids), the $\Delta$ TPR domain (108-367 amino acids), and the $\Delta$ SGS domain (1-284 amino acids) are indicated. $\mathbf{b}$ OsSAPK9 interactions with the TPR and CS domains in yeast. The TPR, CS, SGS, $\triangle T P R$, and $\triangle$ SGS domains are from OsSGT1. AH109 yeast transformants diluted 10,100, 1000, or 10,000 times were plated on synthetic dextrose (SD) medium lacking Trp and Leu amino acids (SD L/T) or SD medium lacking Trp, Leu, His and Ade amino acids (SD - L/T/W/A). c OsSAPK9 bound to the TPR domain of OsSGT1 in the glutathione S-transferase (GST) pull-down assay involving His-OsSAPK9 and GST-TPR. d OsSAPK9 bound to the CS domains of OsSGT1 in the glutathione Stransferase (GST) pull-down assay involving His-OsSAPK9 and GST-CS. e OsSAPK9 interactions with the TPR and CS domains in a bimolecular fluorescence complementation assay. OsSAPK9 was fused to an N-terminal yellow fluorescent protein (NYFP-OsSAPK9), and TPR, CS, SGS, $\triangle$ TPR, and $\triangle$ SGS were fused to C-terminal cyan fluorescent proteins (CCFP-TPR, CCFP-CS, CCFP-SGS, CCFP- $\triangle T P R$, and CCFP- $\triangle$ SGS). f OsSAPK9 interactions with OsHsp90 in the bimolecular fluorescence complementation assay. OsSAPK9 was fused to an N-terminal yellow fluorescent protein (NYFP-OsSAPK9), and OsHsp90 was fused to a C-terminal cyan fluorescent protein (CCFP-Hsp90). $\mathbf{g}$ OsSAPK9 bound to OsHsp90 in the GST pull-down assay involving His-OsSAPK9 and GST-OsHsp90 

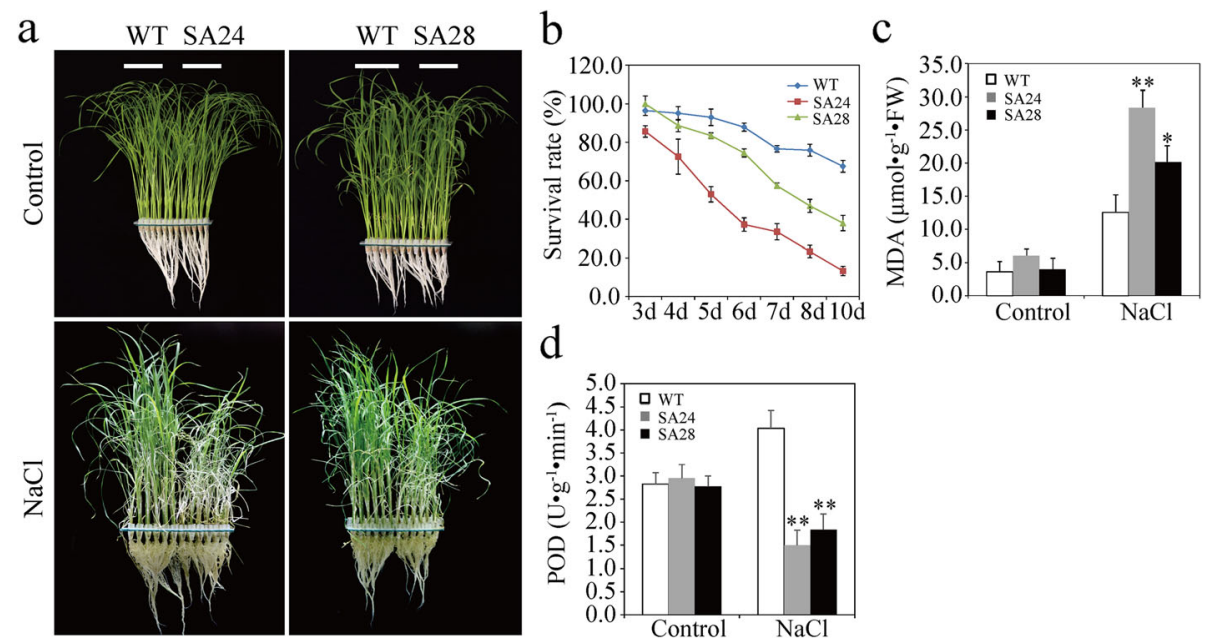

Fig. 5 Phenotypic responses of OsSGT1-overexpression (OsSGT1-OE) and wild-type (WT) plants under salt-stress conditions. a Phenotypes of OsSGT1-OE and WT plants treated with or without $100 \mathrm{mM} \mathrm{NaCl}$. Photographs were taken $6 \mathrm{~d}$ after treatment. b Survival rates of OsSGT1-OE and WT plants under salt-stress conditions (100 mM NaCl). c Malondialdehyde (MDA) contents in OsSGT1-OE and WT plants $6 \mathrm{~d}$ after being treated with $100 \mathrm{mM} \mathrm{NaCl}$. d Peroxidase (POD) activities in OsSGT1-OE and WT plants $6 \mathrm{~d}$ after being treated with $100 \mathrm{mM} \mathrm{NaCl}$. Values are presented as the means and standard errors of three replicates. ${ }^{*} P<0.05$, according to a Student's $t$-test

in the WT plants, while the POD activities were significantly weaker (Fig. 5c, d). Thus, OsSGT1 may negatively regulate rice responses to salt stress. The LLs in the OsSGT1-OE transgenic rice lines were significantly shorter than those of the WT plants following independent inoculations with the Xoo strains GD1358, PXO347, and PXO340 (Fig. 6a-f). However, there were no significant differences in the LLs between the OsSGT1-OE rice lines and the WT plants following inoculation with JS97-2 (Fig. 6g, h). Thus, OsSGT1 appears to positively regulate $\mathrm{Xoo}$ strain-specific resistance in rice.

\section{Transcriptome Profiling of the Defense Responses to Salt} Stress and Xoo Infection Mediated by OsSAPK9

To identify the responses mediated by OsSAPK9, the transcriptome profiles of OsSAPK9-RNAi line Ri-27 and WT plants were compared after exposure to salt stress and inoculation with the Xoo strain GD1358 (Additional file 10: Table S3). Using the criterion of $p<0.05$, 469 differentially expressed genes (DEGs) were identified under salt-stress conditions when the WT was compared with OsSAPK9-RNAi. These included 130, 93, and 121 DEGs at $2 \mathrm{~h}, 6 \mathrm{~h}$, and $24 \mathrm{~h}$, respectively (Fig. 7a; Additional file 11: Table S4). When the plants were inoculated with GD1358, 1389 DEGs were identified in the comparison between WT and OsSAPK9-RNAi; including 91, 106, and 124 DEGs at $2 \mathrm{~h}, 48 \mathrm{~h}$, and $72 \mathrm{~h}$ after inoculation, respectively (Fig. 7b; Additional file 12: Table S5). Among these, 65 DEGs that were significantly enriched in the Gene Ontology (GO) categories negative regulation of peptidase activity $(P=0.00128)$, peptidase inhibitor activity $(P=0.00128)$, terpene synthase activity $(P=$
0.00158), serine-type endopeptidase inhibitor activity $(P=0.00211)$, and defense response $(P=0.00321)$ were detected both under salt-stress conditions and after Xoo inoculation (Fig. 7c-e; Additional file 13: Table S6, Additional file 14: Table S7). Accordingly, there were 404 and 1324 DEGs that were unique to responses to the salt stress and Xoo infection, respectively (Fig. 7c). The DEGs specifically detected under salt-stress conditions were associated with oxidation-reduction process $(F D R=1.27 \mathrm{E}$ $12)$, response to oxidative stress $(F D R=1.10 \mathrm{E}-07)$, fatty acid biosynthetic process $(F D R=7.74 \mathrm{E}-06)$, and response to stress $(F D R=9.33 \mathrm{E}-05)$. (Fig. 7f; Additional file 13: Table S6, Additional file 14: Table S7). Glucan, fatty acids (FAs), and FA-derivatives are important sources of reserve energy and essential components of cell organelles in plants. They also play significant roles in improving stress tolerance in plants by participating in several defense-related pathways. The transcripts $L O C_{-}$ Os03g01800, LOC_Os06g48200, LOC_Os07g36630, LOC_ Os09g25490, LOC_Os10g32980, and LOC_Os11g33270 are involved in glucan metabolic processes and were differentially regulated in this study. LOC_OsO6g48200 and LOC_Os11g33270 were down-regulated in the WT plants in comparison with OsSAPK9-RNAi plants, while the other transcripts were up-regulated in the same comparison (Fig. 7g; Additional file 15: Table S8). There were also 16 DEGs associated with lipid metabolic processes that were up-regulated in the WT plants (Fig. $7 \mathrm{~g}$; Additional file 15: Table S8). Of these processes, redox regulation, antioxidant defense, and ROS signaling are critical in realizing and fine-tuning metabolic activities. There were 43 DEGs associated with the oxidation- 

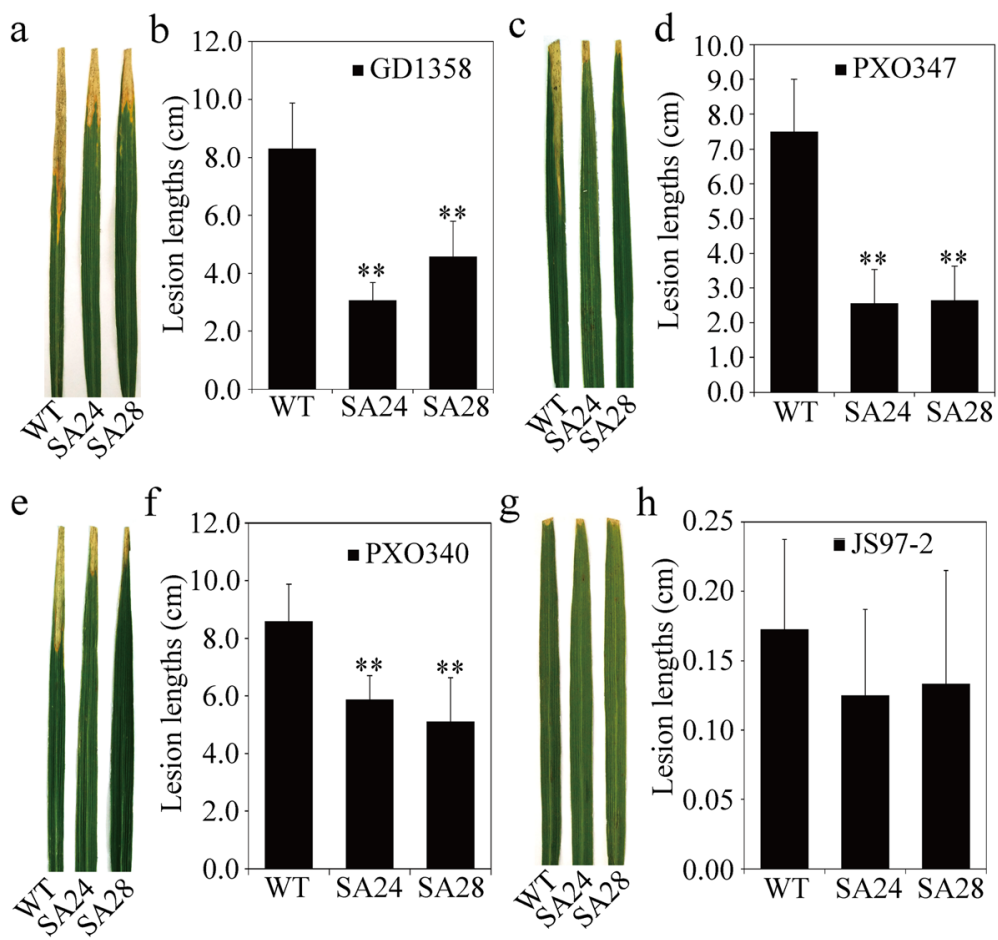

Fig. 6 Phenotypic responses of OsSGT1-overexpression (OsSGT1-OE) and wild-type (WT) plants inoculated with Xanthomonas oryzae pv. oryzae (XOO). a and (b) Phenotypes of OsSGT1-OE and WT plants, respectively, inoculated with Xoo strain GD1358. c and (d) Phenotypes of OsSGT1-OE and WT plants, respectively, inoculated with Xoo strain PXO347. e and (f) Phenotypes of OsSGT1-OE and WT plants, respectively, inoculated with XOO strain PXO340. $\mathbf{g}$ and (h) Phenotypes of OsSGT1-OE and WT plants, respectively, inoculated with XoO strain JS97-2. Photographs were taken $12 \mathrm{~d}$ after inoculation. The lesion lengths were measured $12 \mathrm{~d}$ after inoculation with Xoo strains. Values are presented as the means and standard errors of three replicates. ${ }^{*} P<0.05$, according to a Student's $t$-test

reduction pathway, of which only 8 were downregulated in the WT vs OSSAPK9-RNAi comparison (Fig. 7h; Additional file 15: Table S8). The majority of the 65 DEGs were up-regulated in the WT plants in comparison with the OSSAPK9-RNAi plants at $0 \mathrm{~h}, 2 \mathrm{~h}$, and $6 \mathrm{~h}$, but were down-regulated at $24 \mathrm{~h}$. Thus, the activation of redox and metabolic signaling pathways may be delayed in OsSAPK9-RNAi plants in comparison with WT plants (Additional file 15: Table S8). DEGs induced by Xoo infection were associated with ATP binding (FDR = $1.52 \mathrm{E}-08)$, phosphatidylinositol metabolic process (FDR = 1.43E-05), and similar GO terms (Fig. 7f, Additional file 13: Tables S6, Additional file 14: Table S7). The phosphoinositide phosphate kinases are implicated in membrane trafficking and are important for plant growth, development, and the immune responses (Antignani et al. 2015; Gerth et al. 2017; Gou et al. 2015; Hempel et al. 2017). Six DEGs (LOC Os03g28140, LOC_Os06g14750, LOC_Os08g01390, LOC Os08g34950,LOC_Os09g23740, and LOC_Os12g13440) were enriched in the phosphatidylinositol phosphate kinase activity category, and all of these were down-regulated in the OsSAPK9-RNAi plants at $0 \mathrm{~h}$ and following Xoo inoculation (Fig. 7i; Additional file 16: Table S9). Five DEGs associated with the components of the myosin complex that maintains the stability of the cytoskeleton and cell movement ( $L O C_{-}$ Os02g57190, LOC_Os06g29350, LOC_Os03g48140, LOC_ Os01g51634, and LOC_Os10g19860) were down-regulated in the OsSAPK9-RNAi plants in comparison with the WT plants at $0 \mathrm{~h}$, and these DEGs were up-regulated in the OsSAPK9-RNAi plants after inoculation with Xoo (Fig. 7i; Additional file 16: Table S9). In total, 19 of the 186 DEGs associated with the ATP-binding process were down-regulated in the WT plants in comparison with the OsSAPK9-RNAi plants, while the remainder were up-regulated. Even though most of the 186 DEGs were up-regulated in the OsSAPK9$R N A i$ plants after inoculation with $X o o$, their expression did not significantly change compared with the WT plants inoculated with $X o o$ (Fig. 7j; Additional file 16: Table S9). Thus, the data suggest that the basic life signaling pathways, such as the ATP-binding process, cytoskeleton complex, and phosphatidylinositol phosphate kinase activity, are more rapidly down-regulated in OSSAPK9-RNAi plants than in WT plants after Xoo inoculation.

\section{Discussion}

Plants have evolved cooperative and alternative molecular mechanisms to adapt to adverse environmental conditions. Protein kinases play central roles in signal 


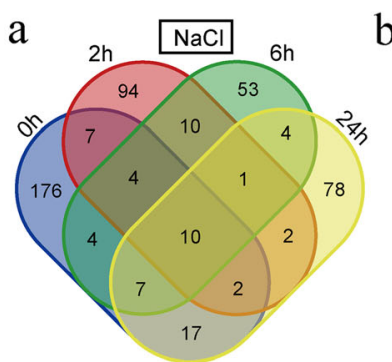

d

- Gene Ontology $\square$ KEGG Pathway $\mathrm{NaCl}$ and $\mathrm{Xoo}$ common DEGs

¿ 2 negative regulation of peptidase activity (BP)

peptidase inhibitor activity (MF)

E 2 terpene synthase activity (MF)

2 serine-type endopeptidase inhibitor activity (MF)

d 4 defense response (BP)

(1) Nitrogen metabolism

$\begin{array}{llll}0 & 2 & 4 & 6\end{array}$

$-\log _{10}(F D R)$

f

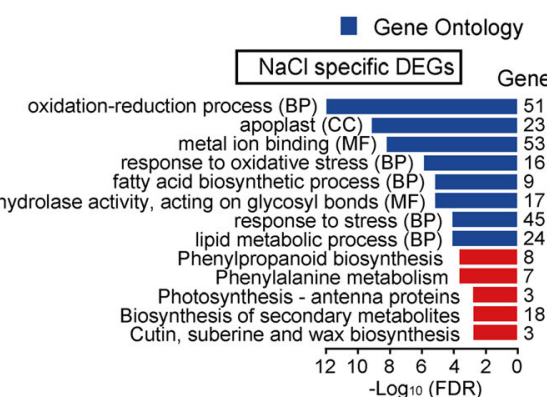

g

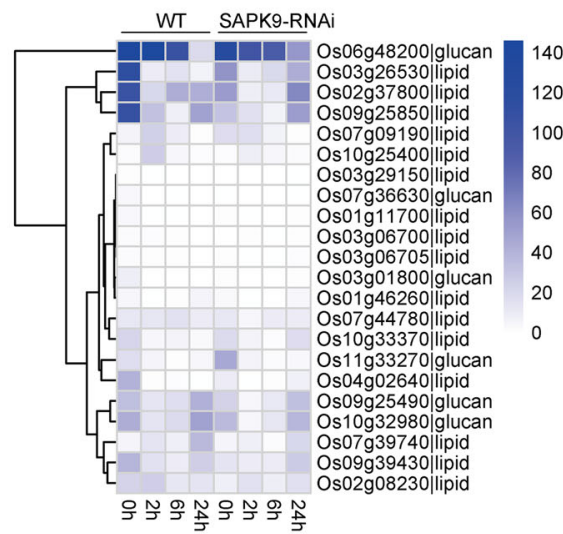

i

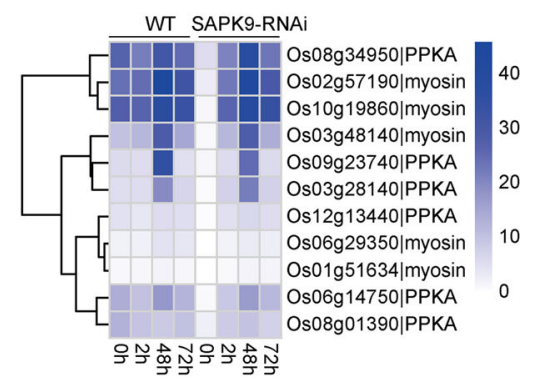

c

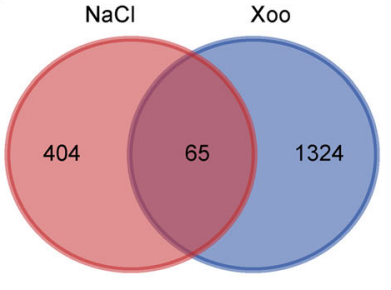

e

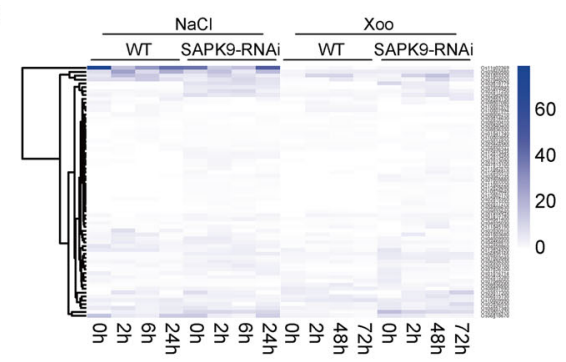

KEGG Pathway

Xoo specific DEGs

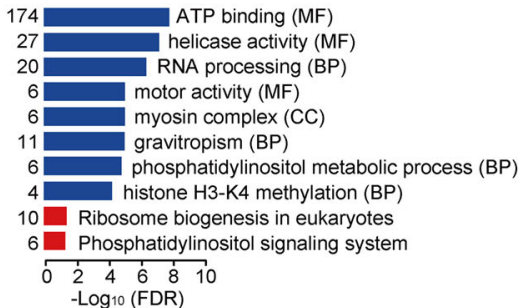

h
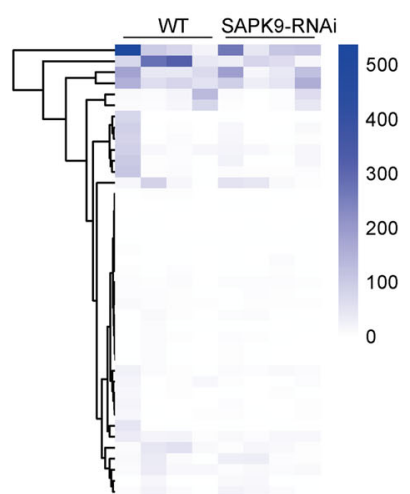

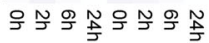

j

WT SAPK9-RNAi

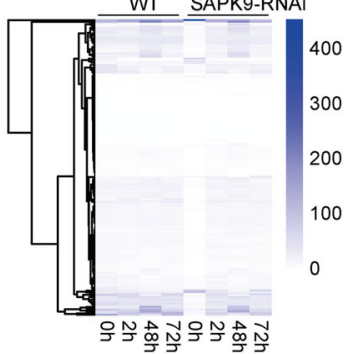

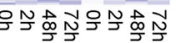

Fig. 7 (See legend on next page.) 
(See figure on previous page.)

Fig. 7 Differentially expressed genes (DEGs) regulated by OSSAPK9 under salt-stress conditions and after inoculation with Xanthomonas oryzae pv. oryzae (Xoo), respectively. a Venn diagram showing the distribution of DEGs in wild-type (WT) vs OsSAPK9-RNAi plants under salt-stress conditions. b Venn diagram showing the distribution of DEGs in WT vs OSSAPK9-RNAi plants after inoculation with Xoo. c Venn diagram showing the distribution of DEGs in WT vS OSSAPK9-RNAi plants under salt-stress conditions and after inoculation with XOO. $\mathbf{d}$ GO and KEGG terms of the 65 common DEGs identified under salt-stress conditions and after inoculation with Xoo. e Hierarchical clustering of the 65 common DEGs under saltstress conditions and after inoculation with Xoo. $\mathbf{f}$ GO and KEGG terms of specific DEGs under salt-stress conditions and after inoculation with XOO, respectively. $\mathbf{g}$ Hierarchical clustering of DEGs in the lipid and glucan metabolic pathways that are regulated by OsSAPK9 under salt-stress conditions. $\mathbf{h}$ Hierarchical clustering of DEGs in the dynamics of the oxidation-reduction pathway that are regulated by OsSAPK9 under salt-stress conditions. i Hierarchical clustering of DEGs in the phosphatidylinositol phosphate kinase activity and the cell cytoskeleton biosynthesis pathway that are regulated by OSSAPK9 after inoculation with XOO. PPKA, phosphatidylinositol phosphate kinase activity. $\mathbf{j}$ Hierarchical clustering of DEGs in the ATP energy signaling pathway that are regulated by OsSAPK9 after inoculation with Xoo

recognition and the subsequent activation of plant defense mechanisms during pathogen infection. The SnRK2s are plant-specific and highly conserved protein kinases that affect responses to various stresses (Fujii et al. 2011; Kulik et al. 2011). In this study, we found that OsSAPK9, which belongs to the $S n R K 2$ gene family, positively regulates salt-stress tolerance and strainspecific resistance to bacterial blight in rice. According to our RNA-seq data, OsSAPK9 regulated the downstream lipid and glucan metabolic pathways in plants after $\mathrm{NaCl}$ treatments, while it mainly regulated the ATP and cell cytoskeleton signaling pathways in plants inoculated with Xoo (Additional file 7: Figure S7). These findings demonstrate the potential of OSSAPK9 as a tool for future crop improvements that may provide dual tolerances to salt and blight stresses. How is OsSAPK9 involved in plant responses to the different stresses associated with saline conditions and pathogen infection? We found that OsSGT1 might play a role in branch regulation by OsSAPK9 in response to abiotic and biotic stresses in rice. The highly conserved eukaryotic cochaperone SGT1 (a suppressor of the G2 allele of $s k p 1$ ) is, in several plant species, a critical protein component of pattern- and effector-triggered immune responses against pathogens (Austin et al. 2002; Azevedo et al. 2002; Hoser et al. 2013; Shi et al. 2015; Tör et al. 2002). In rice, the overexpression of OsSGT1 significantly increases strain-specific basal resistance to Xoo (Wang et al. 2008). Interactions between Hsp90 and SGT1 are required for the accumulation of resistance proteins and for the induction of disease resistance in a wide range of species (Ito et al. 2015; Kadota et al. 2008; Wang et al. 2015). In this study, we confirmed that OsSAPK9 forms a protein complex with OsSGT1 and the molecular chaperone OsHsp90 both in vivo and in vitro. Notably, OsSGT1 positively regulated Xoo strain-specific resistance and negatively regulated the responses to salt stress (Figs. 5 and 6). This finding suggests that OsSGT1 plays a key role in diversifying the function of OsSAPK9 to activate different signaling pathways in response to abiotic and biotic stresses. We speculate that OsSAPK9 positively regulates rice resistance to $\mathrm{Xoo}$ through its interactions with OsSGT1 and OsHsp90. However, the mechanism by which OsSGT1 acts in the signaling pathway mediated by OsSAPK9 in response to salt stress is still obscure. Future research will focus on understanding the genetic relationship between OsSAPK9 and OsSGT1 to further characterize the molecular mechanism through which OsSAPK9 acts in response to diverse stresses.

\section{Conclusion}

In this study, we revealed that OsSAPK9 positively regulates salt-stress tolerance and bacterial blight resistance using OsSAPK9-RNAi and OsSAPK9-overexpression transgenic lines. RNA-seq data indicated that under saltstress conditions and after Xoo inoculation, OsSAPK9 not only regulates the respective specific signaling pathways but also regulates 65 common DEGs involved mainly in defense responses under both treatment conditions. We also revealed that OsSAPK9 interacts with OsSGT1 to regulate rice resistance to bacterial blight and salt stress. These results provide new insights into the mechanisms underlying the OsSAPK9-regulated resistance to biotic and abiotic stresses in rice.

\section{Materials and Methods \\ Vector Construction and Rice Transformation}

OsSAPK9-RNAi plants were created using a previously described RNAi strategy (Qiu et al. 2005). A 553-bp OsSAPK9 cDNA fragment was amplified using PrimeSTAR GXL DNA Polymerase (TaKaRa, Dalian, China) and O. sativa ssp. japonica rice variety $9804 \mathrm{cDNA}$ as the template (primers listed in Additional file 10: Table S2). The primers were specific to the $5^{\prime}$ and $3^{\prime}$ ends of the OsSAPK9 fragment, and included the attB1 and attB2 adaptors, respectively. The resulting amplicon was cloned into the binary vector $p H 7 G W I W G 2(I I)$. To generate the OSSAPK9-OE and OsSGT1-OE lines, the full-length OsSAPK9 and OsSGT1 sequences, respectively, were downloaded from the Gramene database (http://www.gramene.org/) and amplified using PCR with the primers listed in Additional file 9: Table S2. The purified amplification products were independently cloned into the binary 
vector $p M D C 43$ for the subsequent production of a GFPtagged fusion proteins. Gene expression was under the control of the Cauliflower mosaic virus 35S promoter. Rice varieties 9804 and Nipponbare were used to generate the transgenic plants. The above constructs were introduced into Agrobacterium tumefaciens strain EHA105 and incorporated into the genomes of 9804 and Nipponbare plants using an $A$. tumefaciens-mediated transformation method to generate the OSSAPK9-RNAi (Ri), OsSAPK9$O E(\mathrm{OE})$, and OsSGT1-OE (SA) lines. Ri-21 and Ri-27 are homozygous $\mathrm{T}_{3}$ RNAi transgenic lines, and OE1, OE2, SA24, and SA28 are $\mathrm{T}_{2}$ transgenic lines.

\section{PCR and Quantitative Real-Time PCR (qRT-PCR)}

Putative transgenic lines were analyzed by PCR using hygromycin-specific primers (Additional file 9: Table S2). Genomic DNA was isolated from rice leaf samples using cetyl-trimethylammonium bromide as previously described (Saghai-Maroof et al. 1984). Total RNA was extracted from frozen samples using an RNAprep Pure Plant Kit (Tiangen, Beijing, China). The qRT-PCR assays were conducted using a TransScript Two-Step RT-PCR SuperMix (Trans, Beijing, China) with the primers listed in Additional file 9: Table S2. The rice ACTIN2/8 gene was used as an internal control. The qRT-PCR analysis was conducted in triplicate, and the means of three biological replicates were used to represent the gene expression levels.

\section{Southern Blot Analysis}

To estimate the number of copies of T-DNA fragments in the Ri-21 and Ri-27 lines, $15 \mu \mathrm{g}$ genomic DNA was digested with HindIII restriction endonuclease, which did not cut within the T-DNA fragment. The digested DNA was fractionated on a $0.8 \%(\mathrm{w} / \mathrm{v})$ agarose gel, blotted onto nylon membranes, and cross-linked (Sabelli 2007). The membranes were then hybridized with a 768-bp specific digoxigenin-labeled Hyg DNA fragment (Additional file 9: Table S2). Probe labeling and hybridization were conducted using a Digoxigenin-High Prime DNA Labeling and Detection Starter Kit II (Roche, Basel, Switzerland).

\section{Protein Extraction and Immunoblot Analysis}

Proteins were extracted from OsSAPK9-OE and OsSGT1-OE rice seedlings and stored at $-80^{\circ} \mathrm{C}$. Protein extract concentrations were determined using a Bio-Rad Protein Assay Kit (Bio-Rad, CA, USA) with bovine serum albumin as the standard. Protein samples were separated electrophoretically on a $10 \%$ polyacrylamide gel containing protein markers. The proteins were subsequently transferred to a polyvinylidene fluoride membrane (Amersham, London, England) by semi-dry electroblotting (Mini-Protean II system; Bio-Rad). The membrane was blocked with 5\% skim milk and blotted with a commercial GFP-tagged mouse monoclonal antibody (Abmart, Shanghai, China). After extensive washings, the bound primary antibody was detected with horseradish peroxidase-conjugated goat anti-mouse IgG secondary antibody according to the manufacturer's recommendations (Abmart, Shanghai, China). The western blot experiment was repeated at least three times, with essentially the same results.

\section{Phenotypes, MDA Contents, and POD and CAT Activities} of the Transgenic Rice Lines Under Salt-Stress Conditions For the salt treatments, OSSAPK9-RNAi lines Ri-21 and Ri-27, OsSAPK9-OE lines OE1 and OE2, and OsSGT1$O E$ lines SA24 and SA28, and WT were used in this study. The seeds were surface-sterilized in $75 \%(\mathrm{v} / \mathrm{v})$ ethanol for $2 \mathrm{~min}$, 30\% sodium hypochlorite for $30 \mathrm{~min}$, and washed with distilled water for five times, then sowed on 1/2 MS medium (with and without hygromycin) at $25^{\circ} \mathrm{C}$ under $16 \mathrm{~h}$ light $/ 8 \mathrm{~h}$ dark cycles for 3 days. Then, the seedlings of transgenic lines and WT were cultured with Hoagland's solution (Yoshida et al. 1976) in the greenhouse until two-leaf stages, 20-d-old seedlings were transferred to Hoagland's solution supplemented with $100 \mathrm{mM} \mathrm{NaCl}$, and the seedling survival rate was assessed daily. Additionally, 20-d-old seedlings were incubated in Hoagland's solution supplemented with $\mathrm{NaCl}$ for $6 \mathrm{~d}$, and fresh leaves $(0.5 \mathrm{~g})$ were harvested from and used to measure the MDA content and both the POD and CAT activity levels according to the methods described by Alkhateeb et al. (2015) and Shin et al. (2012), respectively.

\section{Artificial inoculation of WT and Transgenic Rice Lines with Xoo}

To evaluate bacterial blight resistance, rice plants were cultivated in a screened house during the natural growing season. Seeds of WT, Ri-21, and Ri-27 plants were sown in a seedling nursery and 30 -d-old seedlings were transferred to the screened house at the Institute of Crop Sciences, Chinese Academy of Agricultural Sciences, Beijing, China. Each line was planted in a singlerow plot with 20 plants in each row $(20 \times 17 \mathrm{~cm})$. Three replicates were used for each line. The transgenic plants (OE1, OE2, SA24, and SA28) were planted in a glasshouse at the Yunnan Academy of Agricultural Sciences, Yunnan, China. At the tillering stage (35 d after transplantation), 4-5 of the uppermost leaves of the plants in a row plot were inoculated with Xoo strains JS97-2, GD1358, PXO340, and PXO347 using the leaf-clipping method (Kauffman et al. 1973). The bacterial isolates were grown on peptone sucrose agar at $30^{\circ} \mathrm{C}$ for $2 \mathrm{~d}$, and the inocula were prepared by suspending the bacteria in sterile water to a final concentration of $10^{8}$ cells $\mathrm{mL}^{-1}$. The LLs were measured on all the inoculated leaves 2 weeks after inoculation, which was when the 
lesions were most obvious and stable. The resistance level of each line was determined using the average LLs of five inoculated plants. Growth curves of the Xoo strain GD1358 in WT, Ri-21, Ri-27, OE1, OE2, SA24, and SA28 plants were produced using the method described by Song et al. (1995). Briefly, more than three leaf fragments per plant were sampled from the plants of all three transgenic lines after inoculation with Xoo. The surfaces of the leaf fragments were sterilized by immersion in $75 \%$ ethanol for $2 \mathrm{~min}$, and then, the leaf fragments were cut and ground in a mortar with $1 \mathrm{~mL}$ sterilized water. The homogenate was diluted to the appropriate volume and $100 \mu \mathrm{L}$ diluted homogenate from each of the samples was spread on PSA solid media. Finally, samples were incubated at $30^{\circ} \mathrm{C}$ for $2 \mathrm{~d}$, and then, the numbers of bacteria on three serial dilution plates that had measurable colony formation were counted.

\section{Plasmid Construction for Protein Expression}

The CDS encoding 361 OsSAPK9 amino acids was amplified using the primers listed in Additional file 10: Table S2. The PCR products were inserted into the $p$ Cold-TF vector to express the histidine-tagged OsSAPK9 protein (HisOsSAPK9) in Escherichia coli (BL21) cells. The CDS encoding 367 OsSGT1 amino acids was amplified as well as the CDSs for the TPR (194 amino acids), CS (172 amino acids), and SGS (113 amino acids) domains using the primers listed in Additional file 9: Table S2. The sequences encoding a 275-amino acid fragment with $\triangle$ SGS and a 260amino acid segment with $\triangle$ TPR were also amplified. The PCR products were cloned into the $p G E X-4 T-1$ vector using an In-Fusion Advantage PCR Cloning Kit (Clontech, Dalian, China) to generate plasmids for the production of the following fusion proteins in E. coli (BL21) cells: GSTOsSGT1, GST-TPR, and GST-CS. The OsHsp 90 CDS was also inserted into the $p G E X-4 T-1$ vector for the production of GST-OsHsp90 proteins.

\section{Yeast Two-Hybrid Assay}

The vectors and yeast strains used in the yeast twohybrid assays were obtained from Clontech. RNA was extracted at specific time points from leaves infected with Xoo strain PXO99A (1-5 d after infection). RNAs were mixed in equal proportions to construct an $\mathrm{AD}$ fusion cDNA library using the Matchmaker System with SMART cDNA synthesis technology (Clontech, Dalian, China). To further verify the interactions between OsSAPK9 and OsSGT1, the corresponding CDSs were amplified using gene-specific primer sets (Additional file 9: Table S2). The full-length OsSAPK9 sequence was cloned into $p G B K T 7$ for the production of $\mathrm{BD}$ OsSAPK9, while the full-length rice OsSGT1 sequence was cloned into $p G A D T 7$ to produce AD-OsSGT1, respectively. Yeast transformation, screening for positive clones, and subsequent reporter gene assays were carried out in accordance with the manufacturer's instructions.

\section{Pull-Down and BiFC Assays}

For the in vitro pull-down assays, full-length OsSAPK9 sequence was inserted into the $p$ Cold-TF vector to produce His-OsSAPK9, and the full-length OSSGT1 and OsHsp 90 sequences were inserted into the $p$ GEX4T-1 vector to produce GST-OsSGT1 and GST-OsHsp90, respectively (Additional file 9: Table S2). The fusion proteins and empty tags (GST or His) were produced in $E$. coli (BL21) cells and purified using the appropriate resin $\left(\mathrm{Ni}^{2+}\right.$ for His tags and GST-binding resin for GST tags). GST-, GST-OsSGT1-, and GST-OsHsp90-coupled beads were used to capture the His tag or His-OsSAPK9. The pull-down assays were completed as described previously (Miernyk and Thelen 2008), and the proteins were detected with horseradish peroxidase-conjugated anti-His monoclonal antibodies (1:1000; Abmart). For the BiFC assay, OsSAPK9 was cloned into the binary BiFC vector $p N Y F P$ (for N-terminal yellow fluorescent protein fusions) to produce NYFP-OsSAPK9, and OsSGT1 and OsHsp 90 were cloned into the binary BiFC vector $p C C F P$ (for C-terminal cyan fluorescent protein $\mathrm{fu}$ sions) to produce CCFP-OsSGT1 and CCFP-OsHsp 90 , respectively (Additional file 9: Table S2). These constructs, as well as the empty vectors, were introduced into $A$. tumefaciens strain EHA105 for the subsequent infiltration of 5-week-old $N$. benthamiana leaves as described by Huang et al. (2018). The leaves were observed $48-72 \mathrm{~h}$ after infiltration using an LSM 700 laser confocal scanning microscope (ZEISS Microsystems, Jena, Germany). The peak excitation wavelengths of YFP was $528 \mathrm{~nm}$.

\section{Co-IP Assay}

For the Co-IP assay, the OsSAPK9 and OsSGT1 CDSs were introduced into the binary vectors $p M D C 43$ (containing the GFP tag) and $p G W B 18$ (containing the Myc tag), respectively. These constructs were inserted into $A$. tumefaciens strain EHA105 for the infiltration of 5week-old $N$. benthamiana leaves as described by Huang et al. (2018). Proteins were extracted from infiltrated leaves $48-72 \mathrm{~h}$ after infiltration. Samples were ground in liquid nitrogen, and proteins were extracted in Co-IP buffer $(50 \mathrm{mM}$ Tris-Cl, pH 7.4, $500 \mathrm{mM} \mathrm{NaCl}, 10 \%$ glycerol, $5 \mathrm{mM}$ EDTA, $1 \%$ Triton X-100, and 1\% Nonidet P-40) containing a protease inhibitor cocktail (Sigma-Aldrich, St. Louis, MO, USA). We used $10 \%$ of the extract as the input control. An agarose-conjugated Myc-tagged mouse monoclonal antibody (Abmart) solution was added to the extract, which was then incubated for $2 \mathrm{~h}$ at $4{ }^{\circ} \mathrm{C}$. Beads were washed five times with Co-IP buffer lacking protease inhibitors. Sodium dodecyl sulfate 
(SDS)-polyacrylamide gel electrophoresis (PAGE) loading buffer $(100 \mathrm{mM}$ Tris-Cl, pH 6.8, 4\% SDS, $0.2 \%$ bromophenol blue, 20\% glycerol, and $200 \mathrm{mM}$ dithiothreitol) was added to the eluted protein samples, which were then boiled for $5 \mathrm{~min}$. We used $25 \%$ of the eluted proteins as the immunoprecipitation control. Anti-GFP and anti-Myc antibodies (Abmart) were used to detect the GFP-OsSAPK9 and MYC-OsSGT1 fusion proteins, respectively.

\section{RNA-seq of Transgenic and WT Lines}

For the OsSAPK9-RNAi-27 and WT lines treated with $100 \mathrm{mM} \mathrm{NaCl}$ or inoculated with Xoo strain GD1358, total RNA was extracted from the rice seedlings grown under $100 \mathrm{mM} \mathrm{NaCl}$ conditions and from 3-cm long seedling leaf tips of the rice seedling inoculated with GD1358 using TRIzol reagent according to the manufacturer's instructions (Invitrogen, Waltham, MA, USA). For each sequencing library, $100 \mathrm{mg}$ of RNA from each replicate was mixed together. The raw sequence data reported in this paper have been deposited in the Genome Sequence Archive (GSA) in the Beijing Institute of Genomics Data Center, Beijing Institute of Genomics, Chinese Academy of Sciences, under accession number PRJCA000702 and are publicly accessible at http://bigd. big.ac.cn/gsa (Wang et al. 2017). The libraries were sequenced by the CapitalBio Corporation (Beijing, China) using an Illumina HiSeq 2000 Sequencing System. Low quality nucleotides $(<\mathrm{Q} 20)$ were trimmed from raw sequences for each sample, and then pair-end reads with either or both ends of lengths $<30 \mathrm{bp}$ were removed using an in-house Perl script. Retained high quality reads were mapped to the Michigan State University Rice Genome Annotation Project database (ftp://ftp.plantbiology.msu.edu/pub/data/Eukaryotic_Projects/o_sativa/annotation_dbs/pseudomolecules/version_7.0) using Bowtie (Langmead et al. 2009; Ouyang et al. 2007). The Cuffdiff module was used to identify DEGs. Chi-square tests were used to identify genes showing statistically significant differences in their relative abundance levels (as reflected by the total counts of individual sequence reads) between two samples using the IDEG6 software and a threshold of $p \leq 0.001$ (Romualdi et al. 2003; Vencio et al. 2003; Ye et al. 2006). Pathway and GO enrichment analyses of rice DEGs were conducted using EXPath 2.0 (Chien et al. 2015). Venn diagrams were constructed using online software (http://bioinformatics. psb.ugent.be/webtools/Venn/).

\section{Accession Numbers \\ OsSAPK9: LOC_Os12g39630; OsSGT1: LOC_ Os01g43540.}

\section{Additional Files}

Additional file 1: Figure S1. OSSAPK9 expression under salt-stress conditions and after inoculation with Xanthomonas oryzae pv. oryzae (Xoo).

Additional file 2: Figure S2. Molecular characterization of OSSAPK9RNAi and OSSAPK9-overexpression (OSSAPK9-OE) transgenic plants.

Additional file 3: Figure S3. OSSAPK9 knock-down increases rice sensitivity to $50 \mu \mathrm{M} \mathrm{NaCl}$ in growth assays.

Additional file 4: Figure S4. Phenotypic responses of OSSAPK9-RNAi, OsSAPK9-overexpression (OsSAPK9-OE), and wild-type (WT) plants inoculated with Xanthomonas oryzae pv. oryzae (Xoo).

Additional file 5: Figure S5. The Coomassie Brilliant blue staining results of a pull-down assay.

Additional file 6: Figure S6. Molecular characterization of OSSGT1overexpression (OsSGT1-OE) transgenic plants.

Additional file 7: Figure S7. Model regulated by OSSAPK9 under saltstress conditions and after inoculation with Xanthomonas oryzae pv. oryzae (Xoo), respectively.

Additional file 8: Table S1. Yeast two-hybrid analysis of proteins interacting with OSSAPK9.

Additional file 9: Table S2. Details of the primers used in this study. (PDF $76 \mathrm{~kb}$ )

Additional file 10: Table S3. The summary of RNA-seq data under saltstress conditions and after inoculation with Xanthomonas oryzae pv. oryzae $\left(X_{00}\right)$.

Additional file 11: Table S4. Differentially expressed genes (DEGs) in wild-type (WT) compared with the OsSAPK9-RNAi line, under salt-stress conditions.

Additional file 12: Table S5. Differentially expressed genes (DEGs) in wild-type (WT) compared with the OsSAPK9-RNAi line after inoculation with Xanthomonas oryzae pv. oryzae (Xoo).

Additional file 13: Table S6. GO terms of differentially expressed genes (DEGs) in wild-type (WT) compared with OsSAPK9-RNAi under saltstress conditions and after inoculation with Xanthomonas oryzae pv. oryzae $\left(\mathrm{XoO}_{\mathrm{O}}\right.$.

Additional file 14: Table S7. KEGG pathways of differentially expressed genes (DEGs) in wild-type (WT) compared with OsSAPK9-RNAi under saltstress conditions and after inoculation with Xanthomonas oryzae pv. oryzae $\left(X_{00}\right)$.

Additional file 15: Table S8. Differentially expressed genes (DEGs) associated with oxidation reduction, and glucan and lipid metabolic processes in rice treated with salt stress.

Additional file 16: Table S9. Differentially expressed genes (DEGs) associated with phosphatidylinositol phosphate kinase activity, myosin complex, and ATP-binding processes in rice inoculated with Xanthomonas oryzae pv. oryzae (Xoo)

\section{Abbreviations}

CAT: Catalase; DEGs: Differentially expressed genes; LLs: Lesion lengths; MDA: Malondialdehyde; POD: Peroxidase; SAPK9: Stress-activated protein kinases 9; SnRK2: Sucrose non-fermenting 1 (SNF1)-related protein kinase 2; Xoo: Xanthomonas oryzae pv. oryzae

\section{Acknowledgments}

We thank Shelley Robison, PhD, and Lesley Benyon, PhD, from Liwen Bianji, Edanz Group China (www.liwenbianji.cn/ac), for editing the English text of a draft of this manuscript.

\section{Authors' Contributions}

YZ designed the experiments; FZ, LH, DZ, and TC performed experimental work; FZ performed transcription data analysis; $Y Z$ and FZ wrote the manuscript. All authors read and approved the final manuscript. 


\section{Funding}

This research was supported by the National Natural Science Foundation of China (Grant Nos. 31771762), the National Key Research and Development Program of China (Project No. 2016YFD0100101), the National High-tech Program of China (No. 2014AA10A603), the Bill \& Melinda Gates Foundation (OPP1130530), and partly supported by the CAAS Agricultural Science and Technology Innovative Program.

\section{Availability of Data and Materials}

Not applicable.

\section{Ethics Approval and Consent to Participate}

Not applicable.

\section{Consent for Publication}

Not applicable.

\section{Competing Interests}

The authors declared that they have no competing interests to this work.

\section{Author details}

'Institute of Crop Sciences/National Key Facility for Crop Gene Resources and Genetic Improvement, Chinese Academy of Agricultural Sciences, 12 South Zhong-Guan-Cun Street, Beijing 100081, China. ${ }^{2}$ Graduate School of Chinese Academy of Agricultural Sciences, 12 South Zhong-Guan-Cun Street, Beijing 100081, China. ${ }^{3}$ School of Agriculture, Yunnan University, Kunming, China.

${ }^{4}$ College of Agronomy, Anhui Agricultural University, Hefei, China.

Received: 11 August 2019 Accepted: 17 October 2019

\section{Published online: 11 November 2019}

\section{References}

Alkhateeb SA, Alkhateeb AA, Mel-D S (2015) In vitro response of date palm (Phoenix dactylifera L.) to K/Na ratio under saline conditions. Biol Res 48:63 Antignani V, Klocko AL, Bak G, Chandrasekaran SD, Dunivin T, Nielsen E (2015) Recruitment of PLANT U-BOX13 and the PI4KB1//2 phosphatidylinositol-4 kinases by the small GTPase RabA4B plays important roles during salicylic acid-mediated plant defense signaling in Arabidopsis. Plant Cell 27:243-261

Austin MJ, Muskett P, Kahn K, Feys BJ, Jones JDG, Parker JE, Parker JE (2002) Regulatory role of SGT1 in early R gene-mediated plant defenses. Science 295:2077-2080

Azevedo C, Sadanandom A, Kitagawa K, Freialdenhoven A, Shirasu K, SchulzeLefert P (2002) The RAR1 interactor SGT1, an essential component of R genetriggered disease resistance. Science 295:2073-2076

Bai J, Mao J, Yang H, Khan A, Fan A, Liu S, Zhang J, Wang D, Gao H, Zhang J (2017) Sucrose non-ferment 1 related protein kinase 2 (SnRK2) genes could mediate the stress responses in potato (Solanum tuberosum L.). BMC Genet 18:41

Cheng C, Wang Z, Ren Z, Zhi L, Yao B, Su C, Liu L, Li X (2017) SCFAtPP2-B11 modulates ABA signaling by facilitating SnRK2.3 degradation in Arabidopsis thaliana. PLoS Genet 13:e1006947

Chien CH, Chow CN, Wu NY, Chiang-Hsieh YF, Hou PF, Chang WC (2015) EXPath: a database of comparative expression analysis inferring metabolic pathways for plants. BMC Genomics 16:S6

Dey A, Samanta MK, Gayen S, Maiti MK (2016) The sucrose non-fermenting 1related kinase 2 gene SAPK9, improves drought tolerance and grain yield in rice by modulating cellular osmotic potential, stomatal closure and stressresponsive gene expression. BMC Plant Biol 16:1-20

Diédhiou CJ, Popova OV, Dietz KJ, Golldack D (2008) The SNF1-type serinethreonine protein kinase SAPK4 regulates stress-responsive gene expression in rice. BMC Plant Biol 8:49

Fujii H, Verslues PE, Zhu JK (2011) Arabidopsis decuple mutant reveals the importance of SnRK2 kinases in osmotic stress responses in vivo. Proc Natl Acad Sci U S A 108:1717-1722

Fujita M, Fujita Y, Noutoshi Y, Takahashi F, Narusaka Y, Yamaguchi-Shinozaki K, Shinozaki K (2006) Crosstalk between abiotic and biotic stress responses: a current view from the points of convergence in the stress signaling networks. Curr Opin Plant Biol 9:436-442

Gerth K, Lin F, Daamen F, Menzel W, Heinrich F, Heilmann M (2017) Arabidopsis phosphatidylinositol 4-phosphate 5 -kinase 2 contains a functional nuclear localization sequence and interacts with alpha-importins. Plant J 92:862-878
Gou JY, Li K, Wu K, Wang X, Lin H, Cantu D, Uauy C, Dobon-Alonso A, Midorikawa T, Inoue K, Sánchez J, Fu D, Blechl A, Wallington E, Fahima T, Meeta M, Epstein L, Dubcovsky J (2015) Wheat stripe rust resistance protein WKS1 reduces the ability of the thylakoid-associated ascorbate peroxidase to detoxify reactive oxygen species. Plant Cell 27:1755-1770

Grondin A, Rodrigues O, Verdouca L, Merlot S, Leonhardt N, Maurel C (2015) Aquaporins Contribute to ABA-Triggered Stomatal Closure through OST1Mediated Phosphorylation. Plant Cell 27:1945-1954

Hempel F, Stenzel I, Heilmann M, Krishnamoorthy P, Menzel W, Golbik R, Helm S, Dobritzsch D, Baginsky S, Lee J, Hoehenwarter W, Heilmann I (2017) MAPKs influence pollen tube growth by controlling the formation of phosphatidylinositol 4,5-bisphosphate in an apical plasma membrane domain. Plant Cell 29:3030-3050

Hoser R, Zurczak M, Lichocka M, Zuzga S, Dadlez M, Samuel MA, Ellis BE, Stuttmann J, Parker JE, Hennig J, Krzymowska M (2013) Nucleocytoplasmic partitioning of tobacco N receptor is modulated by SGT1. New Phytol 200: 158-171

Hu DD, Zhang F, Huang LY, Zhuo DL, Zhang F, Zhou YL, Li ZK (2015) Stressactivated protein kinase OSSAPK2 involved in regulating resistant response to Xanthomonas oryzae pv. oryzae in Rice. Acta Agron Sin 41:1191-1200

Huai JL, Wang M, He JG, Zheng J, Dong ZG, Lv HK, Zhao JF, Wang GY (2008) Cloning and characterization of the SnRK2 gene family from Zea mays. Plant Cell Rep 27:1861-1868

Huang LY, Wang YX, Wang WS, Zhao XQ, Qin Q, Sun F, Hu FY, Zhao Y, Li ZC, Fu BY, Li ZK (2018) Characterization of transcription factor gene OsDRAP1 conferring drought tolerance in rice. Front Plant Sci 9:94

Ito M, Ohnishi K, Hikichi Y, Kiba A (2015) Molecular chaperons and co-chaperons, Hsp90, RAR1, and SGT1 negatively regulate bacterial wilt disease caused by Ralstonia solanacearum in Nicotiana benthamiana. Plant Signal Behav 10: e970410

Kadota Y, Amigues B, Ducassou L, Madaoui H, Ochsenbein F, Guerois R, Shirasu K (2008) Structural and functional analysis of SGT1-HSP90 core complex required for innate immunity in plants. EMBO Rep 9:1209-1215

Kauffman HE, Reddy APK, Hsieh SPY, Merca SD (1973) An improved technique for evaluation of resistance of rice varieties to Xanthomonas oryzae. Plant Dis Rep 57:537-541

Kim MJ, Park MJ, Seo PJ, Song JS, Kim HJ, Park CM (2012) Controlled nuclear import of the transcription factor NTL6 reveals a cytoplasmic role of SnRK2.8 in the drought-stress response. Biochem J 448:353-363

Kobayashi Y, Yamamoto S, Minami H, Kagaya Y, Hattori T (2004) Differential activation of the rice sucrose nonfermenting1-related protein kinase 2 family by hyperosmotic stress and abscisic acid. Plant Cell 16:1163-1177

Kulik A, Wawer I, Krzywińska E, Bucholc M, Dobrowolska G (2011) SnRK2 protein kinases-key regulators of plant response to abiotic stresses. OMICS 15:859-872

Langmead B, Trapnell C, Pop M, Salzberg SL (2009) Ultrafast and memoryefficient alignment of short DNA sequences to the human genome. Genome Biol 10:R25

Lee HJ, Park YJ, Seo PJ, Kim JH, Sim HJ, Kim SG, Park CM (2015) Systemic Immunity Requires SnRK2.8-Mediated Nuclear Import of NPR1 in Arabidopsis. Plant Cell 27:3425-3438

Liu Z, Ge X, Yang Z, Zhang C, Zhao G, Chen E, Liu J, Zhang X, Li F (2017) Genome-wide identification and characterization of SnRK2 gene family in cotton (Gossypium hirsutum L.). BMC Genet 13:e1006753

Lou D, Wang H, Liang G, Yu D (2017) OsSAPK2 confers abscisic acid sensitivity and tolerance to drought stress in Rice. Front Plant Sci 8:993

McLoughlin F, Galvan-Ampudia CS, Julkowska MM, Caarls L, van der Does D, Lauriere C, Munnik T, Haring MA, Testerink C (2012) The Snf1-related protein kinases SnRK2.4 and SnRK2.10 are involved in maintenance of root system architecture during salt stress. Plant J 72:436-449

Meldau S, Baldwin IT, Wu JQ (2011) For security and stability: SGT1 in plant defense and development. Plant Signal Behav 6:1479-1482

Miernyk JA, Thelen JJ (2008) Biochemical approaches for discovering proteinprotein interactions. Plant J 53:597-609

Nino-Liu DO, Ronald PC, Bogdanove AJ (2006) Xanthomonas oryzae pathovars: model pathogens of a model crop. Mol Plant Pathol 7:303-324

Ouyang S, Zhu W, Hamilton J, Lin H, Campbell M, Childs K, Thibaud-Nissen F, Malek RL, Lee Y, Zheng L, Orvis J, Haas B, Wortman J, Buell CR (2007) The TIGR Rice genome annotation resource: improvements and new features. Nucleic Acids Res 35:D883-D887

Qiu S. Adema CM, Lane T (2005) A computational study of off-target effects of RNA interference. Nucleic Acids Res 33:1834-1847 
Romualdi C, Bortoluzzi S, D'Alessi F, Danieli GA (2003) IDEG6: a web tool for detection of differentially expressed genes in multiple tag sampling experiments. Physiol Genomics 12:159-162

Sabelli PA (2007) Southern blot analysis. Mol Biomethods Handb 8:77-87

Saghai-Maroof MA, Soliman KM, Jorgensen RA, Allard RW (1984) Ribosomal DNA spacer-length polymorphisms in barley: Mendelian inheritance, chromosomal location, and population dynamics. Proc Natl Acad Sci U S A 81:8014-8018

Saha J, Chatterjee C, Sengupta A, Gupta K, Gupta B (2014) Genome-wide analysis and evolutionary study of sucrose non-fermenting 1-related protein kinase 2 (SnRK2) gene family members in Arabidopsis and Oryza. Comput Biol Chem 49:59-70

Sharma R, De Vleesschauwer D, Sharma MK, Ronald PC (2013) Recent advances in dissecting stress-regulatory cross talk in rice. Mol Plant 6:250-260

Shi Q, Febres VJ, Jones JB, Moore GA (2015) Responsiveness of different citrus genotypes to the Xanthomonas citri ssp. citri-derived pathogen-associated molecular pattern (PAMP) flg22 correlates with resistance to citrus canker. Mol Plant Pathol 16:507-520

Shin L, Lo JC, Yeh KC (2012) Copper chaperone antioxidant protein1 is essential for copper homeostasis. Plant Physiol 159:1099-1110

Shirasu K (2009) The HSP90-SGT1 chaperone complex for NLR immune sensors. Annu Rev Plant Biol 60:139-164

Soma F, Mogami J, Yoshida T, Abekura M, Takahashi F, Kidokoro S, Mizoi J, Shinozaki K, Yamaguchi-Shinozaki K (2017) ABA-unresponsive SnRK2 protein kinases regulate mRNA decay under osmotic stress in plants. Nat Plants 3: 16204

Song WY, Wang GL, Chen LL, Kim HS, Pi LY, Holsten T, Gardner J, Wang B, Zhai WX, Zhu LH, Fauquet C, Ronald P (1995) A receptor kinase-like protein encoded by the rice disease resistance gene, Xa21. Science 270:1804-1806

Tan W, Zhang D, Zhou H, Zheng T, Yin Y, Lin H (2018) Transcription factor HAT1 is a substrate of SnRK2.3 kinase and negatively regulates ABA synthesis and signaling in Arabidopsis responding to drought. PLOS Genet 14:e1007336

Tör M, Gordon P, Cuzick A, Eulgem T, Sinapidou E, Mert-Turk F, Can C, Dangl JL, Holub EB (2002) Arabidopsis SGT1b is required for defense signaling conferred by several downy mildew resistance genes. Plant Cell 14:993-1003

Vencio RZ, Brentani H, Pereira CA (2003) Using credibility intervals instead of hypothesis tests in SAGE analysis. Bioinformatics 19:2461-2464

Wang GF, Fan RC, Wang XP, Wang DW, Zhang XQ (2015) TaRAR1 and TaSGT1 associate with TaHsp90 to function in bread wheat (Triticum aestivum L.) seedling growth and stripe rust resistance. Plant Mol Biol 87:577-589

Wang Y, Song F, Zhu J, Zhang S, Yang Y, Chen T, Tang B, Dong L, Ding N, Zhang Q, Bai Z, Dong X, Chen H, Sun M, Zhai S, Sun Y, Yu L, Lan L, Xiao J, Fang X, Lei H, Zhang Z, Zhao W (2017) GSA: genome sequence archive. Genomics Proteomics Bioinformatics 15:14-18

Wang YL, Gao MJ, Li Q, Wang LY, Wang JJ, Jeon JS, Qu N, Zhang YL, He ZH (2008) OsRAR1 and OsSGT1 physically interact and function in rice basal disease resistance. Mol Plant-Microbe Interact 21:294-303

Xu MR, Huang LY, Zhang F, Zhu LH, Zhou YL, Li ZK (2013) Genome-wide phylogenetic analysis of stress-activated protein kinase genes in rice (OsSAPKs) and expression profiling in response to Xanthomonas oryzae pv. oryzicola infection. Plant Mol Biol Report 31:877-885

Yan J, Wang P, Wang B, Hsu CC, Tang K, Zhang H, Hou YJ, Zhao Y, Wang Q, Zhao C, Zhu X, Tao WA, Li J, Zhu JK (2017) The SnRK2 kinases modulate miRNA accumulation in Arabidopsis. PLoS Genet 13:e1006753

Ye J, Fang L, Zheng H, Zhang Y, Chen J, Zhang Z, Wang J, Li S, Li R, Bolund L, Wang J (2006) WEGO: a web tool for plotting GO annotations. Nucleic Acids Res 34:W293-W297

Yoshida R, Hobo T, Ichimura K, Mizoguchi T, Takahashi F, Aronso J, Ecker JR, Shinozaki K (2002) ABA-activated SnRK2 protein kinase is required for dehydration stress signaling in Arabidopsis. Plant Cell Physiol 43:1473-1483

Yoshida S, Forno DA, Cock JH, Gomez KA (1976) Laboratory manual for physiological studies of rice. International Rice Research Institute Press, Philippines, pp 61-64

Zheng ZF, Xu XP, Crosley RA, Greenwalt SA, Sun YJ, Blakeslee B, Wang LZ, Ni WT, Sopko MS, Yao CL, Yau K, Burton S, Zhuang MB, McCaskill DG, Gachotte D, Thompson M, Greene TW (2010) The protein kinase SnRK2.6 mediates the regulation of sucrose metabolism and plant growth in Arabidopsis. Plant Physiol 153:99-113

\section{Publisher's Note}

Springer Nature remains neutral with regard to jurisdictional claims in published maps and institutional affiliations.

\section{Submit your manuscript to a SpringerOpen ${ }^{\circ}$ journal and benefit from:}

- Convenient online submission

- Rigorous peer review

- Open access: articles freely available online

- High visibility within the field

- Retaining the copyright to your article

Submit your next manuscript at $\boldsymbol{\nabla}$ springeropen.com 